\title{
La didactique des langues : les relations entre les plans psychologique, linguistique et pédagogique
}

\author{
Claude Germain \\ Département de didactique des langues \\ Université du Québec à Montréal (UQAM) \\ germain.claude@uqam.ca \\ Joan Netten \\ Faculty of Education \\ Memorial University of Newfoundland (MUN) \\ joan.netten@sympatico.ca
}

\begin{abstract}
Jusqu'ici, en didactique des langues, l'étude du plan psychologique a été négligée en regard des plans linguistique et pédagogique: il n'est donc pas étonnant que les stratégies d'enseignement qui en découlent aient été peu développées. Par le passé, l'accent n'a été mis pour ainsi dire que sur les seuls aspects curriculaires du domaine, comme cela a été le cas, par exemple, dans Un niveau-seuil (qui a marqué l'orientation de l'enseignement des langues en milieu canadien) dans les années soixante-dix : en effet, rien n'est mentionné, dans ce volumineux document, sur le fondement de l'acquisition d'une langue seconde ou étrangère (L2/LE), non plus que sur les relations entre la conception de l'acquisition d'une L2/LE et les stratégies d'enseignement qui en découlent. Et, plus récemment, dans le Cadre européen commun de référence pour les langues (2000), au chapitre 6 , on ne trouve qu'une timide tentative de rapprochement entre les deux notions. En règle générale, en didactique des langues, l'accent n'a été mis pour ainsi dire que sur les seuls aspects curriculaires. En effet, nombre de chercheurs se sont concentrés surtout sur l'objet à faire apprendre : la langue (le plan linguistique). C'est pourquoi les stratégies d'enseignement (le plan pédagogique), ainsi que le mode d'acquisition de la langue (le plan psychologique), ont été laissés pour compte. Et en cela, les chercheurs et théoriciens ont été étroitement suivis par les auteurs de manuels mis à la disposition des enseignants de langue. C'est ainsi que la didactique des langues souffre d'un sérieux déséquilibre dont on ne semble pas mesurer l'ampleur de l'impact. Au cours du présent article, nous nous intéresserons donc en particulier aux relations entre les trois plans majeurs de la didactique des langues : les plans psychologique, linguistique et pédagogique. Dans la première partie, nous ferons d'abord état de la conception de l'enseignement de la langue qui se dégage à la fois des manuels utilisés et de nos observations de classe; puis, de la conception de la langue qui est sous-jacente à cette conception de l'enseignement; et enfin, de la conception de l'acquisition de la langue qui est sous-jacente à ces deux conceptions. Dans la deuxième partie, nous procéderons dans l'ordre inverse : nous montrerons comment une autre conception de l'acquisition mène à une conception véritablement communicative de la langue, et partant, à un enseignement véritablement communicatif d'une L2/LE.
\end{abstract}

\section{Première partie}

Dans la première partie, nous ferons d'abord état de la conception courante de l'enseignement d'une L2/LE; puis, nous montrerons que cette conception découle de la conception implicite que les enseignants se font de la langue, laquelle découle elle-même de la conception qu'ils ont de son mode d'acquisition. En d'autres mots, la façon d'enseigner la langue est tributaire de la conception que l'on se fait de la langue, cette dernière découlant elle-même de la conception que l'on se fait de son mode d'acquisition. 


\subsection{Conception courante de l'enseignement de la langue}

Avant d'examiner une conception courante de l'enseignement de la langue, il importe de préciser qu'au Canada, depuis de très nombreuses années, les deux principaux régimes pédagogiques destinés à l'apprentissage du français langue seconde (FLS) sont : l'immersion et le français de base. L'immersion (précoce, moyenne ou tardive) consiste avant tout en l'apprentissage en français (au fil des ans) des matières scolaires, comme les mathématiques, les sciences, les sciences humaines ou sociales, etc., ce qui signifie que l'élève doit apprendre simultanément le FLS et certaines matières. Quant au français de base, qui débute le plus souvent en $4^{\mathrm{e}}$ année (élèves de 9 ans environ), il se caractérise par de brèves périodes, non successives, de $40-45$ minutes par jour destinées à l'apprentissage du FLS, ce qui représente un total d'environ 90 heures par année scolaire ; c'est ce que nous appelons la méthode « au comptegouttes » (drip-feed method). Or, au Canada, environ 85\% des élèves qui apprennent le FLS sont en français de base et $15 \%$ en immersion. En règle générale, les élèves de l'immersion réussissent à communiquer avec aisance et spontanéité en français et développent, ainsi, une grande autonomie langagière (malgré des failles reconnues quant à la précision langagière). Par contre, même après plusieurs années d'apprentissage, comme on le verra ci-dessous à l'aide de données empiriques, la très grande majorité des élèves anglophones canadiens du français de base n'arrivent toujours pas à communiquer en français et ne développent pas un niveau satisfaisant d'autonomie langagière : ils ne recourent qu'à des phrases ou formules stéréotypées, n'ont pas d'initiative langagière et ne peuvent utiliser la langue avec spontanéité dans des situations authentiques de communication.

Ainsi, au Canada, à quelques exceptions près, la très grande majorité des enseignants de FLS sont en français de base. Dans le cadre de ce régime pédagogique, nous avons pu identifier un certain nombre d'activités pédagogiques et de stratégies courantes d'enseignement (Germain et Netten, à paraître) ${ }^{1}$. Par exemple ${ }^{2}$,

- faire mémoriser des listes de mots de vocabulaire sur les voitures (le pare-brise, les essuie-glaces, la ceinture de sécurité, etc.);

- faire associer des mots de vocabulaire à différentes illustrations (un poisson, une tortue, une étoile, etc.);

- faire associer, comme activité de " production orale », des illustrations à une liste d'expressions écrites (comme Vous permettez? - Oui, merci.);

- proposer des exercices à trous afin de faire utiliser les partitifs appropriés $d u$, de la, de l', des (Qu'est-ce qu'il y a au menu? - Il y a... salade de tomates ou... saucisson);

- faire conjuguer des verbes (par exemple, conjuguer les verbes être, avoir, faire, aller et venir au futur antérieur de l'indicatif, ou encore, «s'exprimer au passé »);

- proposer une « grammaire en dialogues »;

- proposer une « grammaire expliquée du français » (par exemple, la différence entre les auxiliaires être et avoir);

- proposer des tableaux grammaticaux (par exemple, un tableau sur l'article défini, un tableau sur l'adjectif interrogatif quel, etc.);

- proposer des activités de réinvestissement de ce qui a été appris au cours d'une unité pédagogique;

- proposer des vrai ou faux;

- dans la très grande majorité des cas, l'enseignant écrit au tableau les nouveaux mots de vocabulaire afin que l'élève puisse les reconnaître au moment de lire et qu'il puisse éventuellement les écrire correctement.

Il faut cependant préciser que la plupart des activités pédagogiques et des stratégies d'enseignement utilisées en salle de classe sont inspirées des manuels ou guides pédagogiques employés en français de base au Canada.

Mais, ce qu'il faut surtout remarquer est que toutes ces activités et stratégies visent à l'apprentissage d'un savoir explicite sur la langue, alors qu'on vise en réalité à la communication, ou à la capacité d'utiliser la langue dans des situations authentiques de communication. Le savoir explicite réfère au savoir qui peut être énoncé ou exprimé, dont une personne est consciente : par exemple, énoncer la règle de l'accord du participe passé avec avoir, réfère à un savoir explicite. À l'inverse, le fait de mettre l'adjectif épithète au bon endroit (en français, on dit : C'est un gros ballon rouge et non C'est un rouge gros ballon ou C'est un 
rouge ballon gros), sans contrôle conscient, c'est-à-dire sans être en mesure d'en énoncer la règle, réfère à une compétence implicite, non consciente. Et l'un (le savoir) peut exister sans l'autre (l'habileté) et vice-versa. Ainsi, sur le plan linguistique, afin de parvenir à faire communiquer l'élève, on semble croire qu'il faille d'abord établir de solides bases portant sur le savoir langagier (lexique et grammaire) et que, suite à une série d'exercices, ce savoir va pouvoir se transformer en habileté à communiquer. Autrement dit, le conscient (ou savoir explicite), grâce à des exercices langagiers, va se transformer en non conscient (ou compétence implicite).

\subsection{Conception sous-jacente de la langue}

Dans cette perspective, la langue est d'abord enseignée en tant qu'un objet d'études scolaires (en tant que savoir) plutôt qu'en tant que moyen de communication. En abordant l'acquisition de la langue comme on aborde l'acquisition des autres matières scolaires, sans s'en rendre compte, les enseignants de langue traitent cette dernière comme si elle était déjà connue. Par exemple, quand on enseigne les mathématiques en français à des élèves francophones, on présuppose, en quelque sorte, que le français est suffisamment connu pour pouvoir comprendre les concepts mathématiques. Les mathématiques sont ainsi abordées, au moyen de la langue, en tant qu'objet d'études scolaires. De la même manière, lorsque la langue est abordée par le biais de l'apprentissage d'un savoir sur la langue, cette dernière est enseignée en tant qu'objet d'études scolaires. Et cet objet comporte deux dimensions : savoir grammatical, sous la forme de règles de grammaire et/ou de verbes à conjuguer, et savoir lexical.

Dans l'ordre du savoir grammatical, on fait généralement conjuguer des verbes ou mémoriser des règles de grammaire ou des dialogues pour faire apprendre à communiquer oralement : la langue devient ainsi un objet d'étude grammaticale. L'accent est donc mis sur la forme (focus on form) plutôt que sur l'utilisation de la langue (focus on use). En effet, faire conjuguer des verbes ou faire mémoriser des règles de grammaire ou des dialogues réfère à un savoir explicite sur la langue, qui est la façon habituelle d'apprendre la plupart des autres matières scolaires. Or, le fait de procéder dans l'apprentissage d'une langue de la même manière que dans l'apprentissage des autres matières scolaires produit un glissement : on glisse d'une conception de la langue en tant que moyen de communication vers une conception de la langue en tant qu'objet d'étude grammaticale.

Dans l'ordre du savoir lexical, on fait généralement mémoriser des listes de mots de vocabulaire pour faire apprendre à communiquer oralement, c'est-à-dire des listes de mots sans lien entre eux (sauf le fait, parfois, de relever d'une même thématique). Or, le fait de mettre ainsi l'accent sur le vocabulaire, en début d'apprentissage d'une langue, produit un second glissement de conception. La langue devient, subrepticement, un moyen de description du réel. C'est pourquoi beaucoup d'enseignants recourent fréquemment à la question $Q u$ 'est-ce que c'est?, dont la réponse ne peut consister qu'en un énoncé descriptif C'est un/une... plutôt qu'en un énoncé véritablement communicatif.

Pourtant, l'apprentissage d'une langue à des fins de communication n'est pas de la même nature que l'apprentissage des autres matières scolaires, la langue n'étant pas un objet d'études scolaires comme les autres matières, mais bien un moyen qui peut servir, précisément, à faire acquérir ces matières. Il y a ici un glissement de conception dont peu d'enseignants semblent être conscients. Il y a plus. La plupart des manuels existants proposent de nombreux exercices, reposant en très grande partie sur la langue écrite (les exercices à trous, les vrai ou faux, les questions à choix multiples, etc.), alors qu'il s'agit de faire acquérir d'abord la langue orale, avant la lecture et l'écriture. La plupart des exercices ont aussi pour but de vérifier l'acquisition du savoir, lexical et grammatical, sur la langue.

En somme, en règle générale, d'après les activités pédagogiques et les stratégies d'enseignement utilisées dans leur salle de classe, les enseignants paraissent présupposer qu'il faut d'abord faire acquérir un savoir sur la langue avant de faire utiliser celle-ci et présupposer également que l'élève est en mesure de lire et d'écrire dans la langue qu'il est en train d'apprendre. Il est intéressant de noter que les préfaces et les introductions des manuels utilisés affirment qu'une langue est, bien entendu, un moyen de communication. Or, si l'on examine de près le contenu et la démarche de ces manuels, on se rend vite 
compte que la conception sous-jacente que l'on se fait de la langue est qu'elle est beaucoup moins un moyen de communication qu'un objet d'étude grammaticale ou de description du réel. De plus, c'est la langue écrite qui sert avant tout de support à l'apprentissage de la langue orale. Et dans ce cas, on n'enseigne pas vraiment aux élèves à lire et à écrire : on présuppose qu'ils le savent déjà sous prétexte qu'ils savent déjà lire et écrire dans leur langue première mais, cela ne signifie pas pour autant qu'ils peuvent le faire dans leur L2/LE. Comme le matériel utilisé oriente, à leur insu, la conception que les enseignants se font de la langue, on comprend que, pour plusieurs d'entre eux, une langue soit davantage traitée comme un objet d'étude grammaticale ou de description du réel' ${ }^{3}$.

\subsection{Conception sous-jacente de l'acquisition d'une L2/LE}

Maintenant, sur le plan de l'acquisition d'une L2/LE, il convient vraisemblablement de faire une distinction entre la conception des auteurs de manuels et des enseignants de langue, d'une part, et la conception des chercheurs spécialistes de l'acquisition d'une L2/LE, d'autre part. En effet, il est fort probable que la plupart des auteurs de manuels et des enseignants de langue ne soient pas conscients du fait que, pour faire apprendre à communiquer dans une L2/LE, il faut d'abord assurer le développement, chez l'élève, d'une compétence implicite. Par contre, chez les chercheurs ou les théoriciens, spécialistes de l'acquisition, on fait couramment la distinction entre compétence implicite et savoir explicite mais, la conception la plus courante, chez la plupart des spécialistes, est que la savoir peut effectivement se tranformer en habileté, à l'aide d'exercices. En effet, lorsqu'on examine de près les théories actuelles de l'acquisition d'une $\mathrm{L} 2 / \mathrm{LE}^{4}$, on constate que la plupart ne font que cautionner, en quelque sorte, les pratiques courantes des enseignants. Par exemple, selon des auteurs comme Anderson (1990) et DeKeyser (1998), l'acquisition d'une matière scolaire repose sur la distinction entre mémoire déclarative (la connaissance des faits) et mémoire procédurale (comment accomplir une tâche cognitive). De cette distinction, on induit que le développement de l'habileté à communiquer implique un processus de procéduralisation, par exemple, à l'aide d'exercices, du savoir appris de manière déclarative. L'une des caractéristiques de ce modèle est que le savoir déclaratif se transforme en savoir procédural, par exemple, à l'aide d'exercices, sans qu'il y ait perte du savoir déclaratif. Autrement dit, cela revient à croire qu'un savoir, à l'aide d'exercices, peut se transformer en habileté. En outre, on semble croire que le savoir est nécessaire pour développer l'habileté à communiquer (Painchaud et Boyko, 1990).

Mais, qu'il s'agisse des auteurs de manuels ou des enseignants, d'une part, ou des chercheurs, d'autre part, si on examine la conception sous-jacente de l'ordre dans lequel on fait acquérir ce qui est nécessaire pour communiquer à l'aide d'une langue, on constate qu'elle comporte une triple confusion :

- confusion dans l'ordre d'acquisition d'un savoir (explicite) sur la langue et de l'habileté à communiquer (ou compétence implicite)

- confusion dans l'ordre d'acquisition de la grammaire explicite (consciente) et de la grammaire interne (non consciente)

- $\quad$ confusion dans l'ordre d'acquisition de l'écrit et de l'oral.

\section{Confusion dans l'ordre d'acquisition d'un savoir (explicite) et d'une habileté (implicite)}

On croit généralement que l'acquisition d'un savoir (explicite) doit précéder le développement de l'habileté à communiquer (ou compétence implicite). Et, comme on vient de le voir, le savoir à acquérir peut consister en un savoir grammatical (des règles de grammaire ou la conjugaison des verbes) ou en un savoir lexical (des listes de mots de vocabulaire hors contexte). Et, dans ces deux cas, il s'agit de savoirs qui, croit-on, doivent être acquis avant de pouvoir développer l'habileté à communiquer, ou afin de parvenir à développer cette habileté. Puis, on semble croire généralement que ces informations éparses sur la langue, à l'aide d'une série d'exercices, vont se transformer en habileté à communiquer.

\section{Confusion dans l'ordre d'acquisition de la grammaire explicite (consciente) et de la grammaire interne (non consciente)}

En faisant apprendre explicitement des règles de grammaire et la conjugaison des verbes, on croit que l'acquisition d'un savoir explicite, conscient de la langue (la grammaire), doit précéder l'acquisition de la 
compétence implicite, c'est-à-dire la construction non consciente par l'élève de sa grammaire interne, dans sa tête (Paradis, 1994, 2004 et 2009). On croit donc que le conscient (la grammaire externe) doit précéder le non conscient (la compétence implicite), car c'est le conscient, croit-on, qui doit être procéduralisé. On semble oublier que la grammaire interne, construite dans la tête de l'élève, pourrait être effectivement très différente de la grammaire externe, c'est-à-dire des manuels de grammaire ou des tableaux grammaticaux habituellement mis à la disposition des élèves. Selon certains chercheurs (Ellis, 1994; Paradis 2004, 2009), la compétence implicite pourrait même être constituée d'un réseau de connexions neuronales fréquentes plutôt que de règles proprement dites. Si tel était le cas, on comprend mieux comment des exercices langagiers ne pourraient pas réussir à transformer un savoir explicite en une compétence implicite, faite de connexions neuronales fréquentes.

\section{Confusion dans l'ordre d'acquisition de l'écrit et de l'oral}

En proposant aux élèves des exercices, écrits pour la plupart, sous diverses formes (exercices à trous, etc.) afin de leur faire apprendre à communiquer oralement, on semble croire que l'acquisition de l'écrit doit précéder l'acquisition de l'oral. De la même manière, en écrivant au tableau les nouveaux mots de vocabulaire introduits en salle de classe, on semble croire que le support de l'écrit est nécessaire au développement de l'oral et le précède.

L'ensemble des activités pédagogiques proposées aux élèves dans les manuels ainsi que les stratégies utilisées par les enseignants dans leur salle de classe reflètent, du point de vue de l'acquisition d'une L2/LE, un ordre d'acquisition des éléments langagiers qui repose avant tout sur la croyance qu'un savoir explicite sur la langue doit précéder l'habileté à communiquer et que, par exemple, à l'aide d'une série d'exercices, écrits pour la plupart, le savoir (explicite) sur la langue va se transformer en habileté à communiquer (ou compétence implicite), que le conscient va se transformer en non conscient.

On justifie généralement l'utilisation des exercices et des stratégies d'enseignement décrits ci-dessus en soutenant qu'à l'aide de ces exercices et de ces stratégies, l'élève pourra éventuellement réussir à assembler tous les éléments langagiers dans le bon ordre afin de parler ou de communiquer dans la langue à apprendre. En effet, selon une opinion largement répandue, pour faire acquérir une langue en tant que moyen de communication, on croit qu'il faut suivre trois étapes, dans cet ordre :

1. savoir explicite sur la langue : mémorisation de mots de vocabulaire, conjugaison de verbes et, dans certains cas (selon l'âge des élèves), étude des règles de grammaire;

2. exercices afin, croit-on, de vérifier que le savoir explicite est correct;

3. compétence implicite : activités de communication afin, croit-on, de transformer le savoir explicite en compétence implicite.

Telle semble être, en tout cas, la conception du mode d'acquisition de la langue que se font bon nombre d'enseignants en français de base, vraisemblablement influencés par la conception sous-jacente de l'acquisition de la langue qu'ont les auteurs des manuels utilisés.

Tout porte donc à croire que la conception que l'on se fait de l'acquisition de la langue influe, en quelque sorte, sur la conception que l'on se fait de la langue : et même si les auteurs de manuels et les enseignants de langue affirment que la langue est d'abord et avant tout un moyen de communication, elle est effectivement traitée comme un objet d'étude grammaticale et comme un objet de description du réel, et on privilégie la forme écrite alors qu'il s'agit de faire acquérir d'abord l'habileté à communiquer à l'oral. Ainsi, le plan psychologique (conception de l'acquisition de la langue) paraît être à la source d'une transformation sur le plan linguistique. Par ricochet, c'est cette conception de la langue qui oriente les stratégies d'enseignement utilisées dans la salle de classe, tel que mentionné précédemment.

\section{Résultats à l'oral en français de base}

Il nous apparaît que la conception de l'acquisition d'une L2/LE, telle que rapportée ci-dessus, tend à faire adopter des stratégies d'enseignement non véritablement communicatives et ne produit pas les résultats escomptés (Netten et Germain, 2009). C'est ainsi qu'afin de mesurer l'efficacité du français de base, en 
production orale, nous avons procédé à son évaluation dans la plupart des provinces et territoires canadiens, à partir de 2002. À cette fin, un test d'entrevue orale individuelle a été administré à un grand nombre d'élèves par des spécialistes des provinces et territoires, tous formés par une même personne et recourant à un même instrument de mesure : l'échelle MSS - New Brunswick Middle School Scale (pour plus de détails sur cette échelle, voir Netten et Germain, 2009).

Tableau 1

Niveaux de l'échelle New Brunswick Middle School Scale.

$\mathrm{U}$ (Uncotable) : aucun emploi de la L2

NL (Novice Low) : mots isolés

NM (Novice Mid) : bribes de phrases

BL (Basic Low) : début de communication spontanée, en phrases complètes

BM (Basic Mid) : concept du «passé »

BH (Basic High) : concept du « futur » et phrases complexes

NG (Novice High) : bribes de phrases plus longues

I (Intermediate) : communication spontanée sur une grande variété de sujets

Les résultats des pré-tests administrés entre 2002 et 2006 à environ 700 élèves montrent que ceux-ci sont incapables de communiquer avec spontanéité après quatre ans d'apprentissage " au compte-gouttes », c'est-à-dire après 360 heures extensives (Figure 1).

Figure 1- Français de base: production orale, niveau atteint après quatre ans (360 heures extensives), début de la $5^{\mathrm{e}}$ année (élèves : 10 ans), 31 classes, 2002-2006 $(\mathrm{N}=689)$

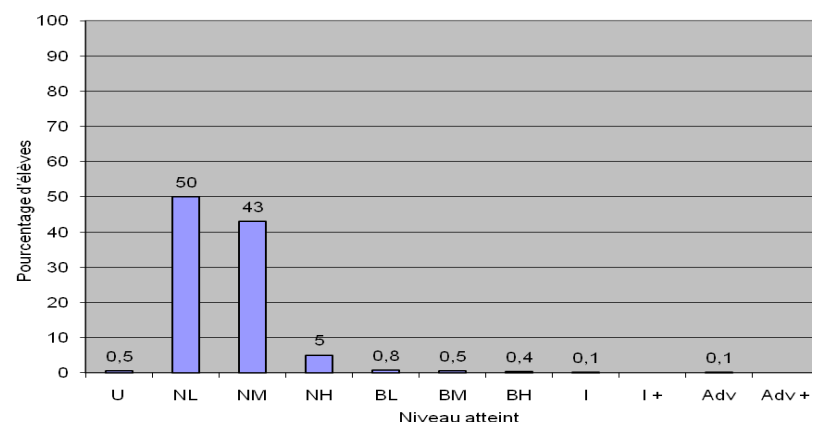

Mais, il y a plus. Nos recherches empiriques montrent que, quel que soit le nombre d'années d'apprentissage de la langue en français de base, les résultats sont toujours insatisfaisants (Figure 2).

Figure 2 - Français de base: production orale, niveau atteint après $1,2,3,4,5,6,8$ et 9 ans, 4 provinces/territoires, 77 classes, 2003-2008 $(\mathrm{N}=1642)$

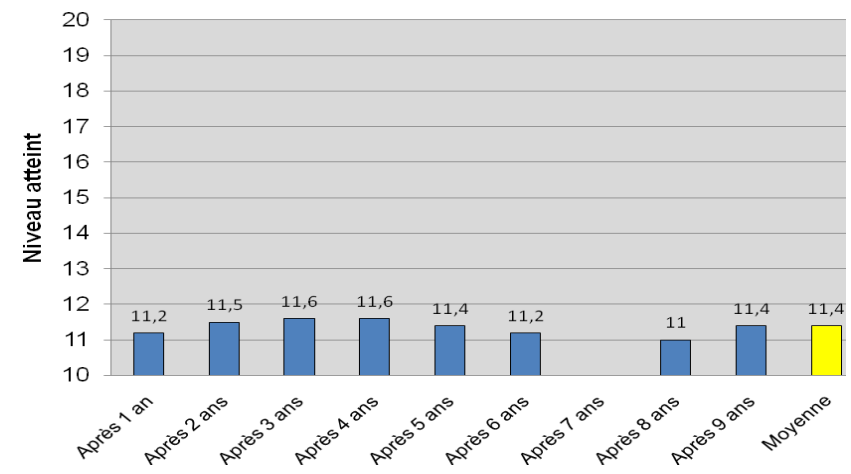


Les piètres résultats obtenus par les élèves inscrits en français de base s'expliquent en partie par l'insuffisance du nombre d'heures et le manque d'intensité de l'enseignement. Mais, selon nous, le principal facteur de l'inefficacité du français de base est le recours à des stratégies d'enseignement qui découlent de la conception sous-jacente que se font les auteurs du matériel pédagogique utilisé pour l'enseignement du FL2/FLE en milieu scolaire.

\section{Deuxième partie}

Ces considérations étant faites, nous pouvons maintenant montrer comment il serait productif de modifier radicalement la façon dont on enseigne une L2/LE en recourant à d'autres stratégies d'enseignement qui reposent sur une autre conception de l'acquisition de la langue menant, à son tour, à une conception véritablement communicative de la langue. Autrement dit, en recourant à une conception radicalement différente de l'acquisition d'une L2/LE, c'est-à-dire en nous référant à une théorie différente de l'acquisition, il parait possible de modifier, en quelque sorte, la conception que se font les enseignants d'une langue et, en fin de compte, de modifier leur façon de l'enseigner, de manière à réussir à faire communiquer leurs élèves.

\subsection{Une autre conception de l'acquisition d'une L2/LE}

Le rôle du savoir explicite dans le développement de la compétence orale en L2/LE est un sujet de débat chez les chercheurs spécialisés en acquisition de la langue. Certaines données empiriques sur le sujet ont montré l'inefficacité du savoir explicite lorsqu'il s'agit de communication orale en L2/LE (Krashen, 1981; Pienemann, 1989; Prabhu, 1987; Truscott, 1999); d'autres chercheurs ont tenté de montrer que le savoir explicite peut avoir certains effets bénéfiques sur le développement de la compétence orale (DeKeyser, 1998; Ellis, 2002; Lightbown et Spada, 1999; Nassaji et Fotos, 2004; Schmidt, 1990; VanPatten, 2002; White et Ranta, 2002 par exemple) ${ }^{6}$. Comme on le verra ci-dessous, nos propres recherches empiriques, entreprises dans le cadre du régime pédagogique du français intensif que nous avons mis au point et implanté en mileu scolaire dès 1998, nous permettent d'adopter le point de vue suivant lequel le savoir explicite ne contribue pas de manière substantielle au développement de la communication orale spontanée. D'ailleurs, sur cette question controversée, White et Ranta (2002) ont pu identifier cinq positions nuancées chez les acquisitionnistes. Comme il serait trop long d'exposer ici les tenants et aboutissants de ce débat (pour plus de détails, voir Netten et Germain, 2005), nous nous contenterons de ne présenter succinctement que la position de Paradis.

En effet, si la plupart des chercheurs paraissent s'entendre sur le fait que, pour pouvoir communiquer dans une langue, il est nécessaire de faire appel à une compétence implicite (ou grammaire interne), rares sont ceux qui sont en mesure d'expliquer comment, dans une salle de classe, il est possible de développer cette compétence implicite. En particulier, comment peut-on rendre compte, dans le cadre d'une seule et même théorie, du fait qu'un élève peut parler sans connaître les règles de la langue et que, inversement, un élève peut connaître les règles de la langue, c'est-à-dire avoir du succès aux examens, sans pouvoir la parler? À notre connaissance, seule la théorie de Paradis (1994, 2004 et 2009) permet de rendre compte de ce double phénomène. C'est pourquoi il importe, afin de mieux comprendre la nature des apprentissages d'une L2/LE, de nous référer ici à la théorie neurolinguistique du bilinguisme de Paradis.

Partant de la distinction bien connue des chercheurs entre la mémoire déclarative et la mémoire procédurale, il ressort des recherches en neurolinguistique qu'il existe une stricte séparation entre ces deux sortes de mémoire, localisées dans deux parties tout à fait distinctes du cerveau, de sorte que la perte de l'une n'entraîne pas nécessairement la perte de l'autre. C'est, en tout cas, ce que révèle l'observation de certaines personnes bilingues atteintes d'aphasie ou de la maladie d'Alzheimer. En effet, la mémoire déclarative, consciente, est associée au savoir (explicite) sur la langue, alors que la mémoire procédurale, non consciente, est plutôt associée à l'utilisation de la langue en tant qu'habileté à communiquer (ou compétence implicite). C'est ce qui paraît susceptible d'expliquer comment une personne bilingue aphasique (dont la compétence implicite a été affectée) peut retrouver plus rapidement ou exclusivement l'usage de la langue qu'elle parlait le moins bien avant sa maladie : même si une personne bilingue 
aphasique peut avoir perdu l'usage d'une partie de l'une des deux langues qu'elle parlait, elle peut encore avoir accès à sa connaissance métalinguistique, comme mécanisme de compensation, précisément parce que cette sorte de connaissance n'a pas été affectée parce qu'elle est localisée ailleurs dans le cerveau. Il se peut que cette connaissance métalinguistique ait été plus développée (en milieu scolaire, par exemple) dans la langue la moins bien acquise sur le plan de l'utilisation dans la vie de tous les jours.

Et, contrairement à une opinion largement répandue chez les enseignants de langue, ce qui est emmagasiné dans la mémoire déclarative, consciente, ne peut être procéduralisé, c'est-à-dire devenir non conscient : le savoir ne peut se transformer en habileté et le conscient ne peut devenir non conscient. Ainsi, le savoir explicite, de nature qualitativement différente de la compétence implicite, ne peut être utilisé que de manière consciente ou délibérée : il ne saurait être utilisé en tant qu'élément du processus de production automatique des énoncés dans une situation authentique de communication. Il ne saurait donc être question d'automatiser (c'est-à-dire d'utiliser de manière non consciente) ou de procéduraliser la connaissance explicite, encore moins de la convertir ou de la transformer en connaissance procédurale : «le processus d'acquisition ne consiste pas en une automatisation de règles dont l'apprenant est conscient, mais bien en l'automatisation de procédures implicites computationnelles (dont l'apprenant n'est pas conscient), sous-jacentes à la compréhension et à la production automatique des énoncés » (Paradis, 1994 - traduction libre). Autrement dit, ce n'est pas la connaissance explicite qui est procéduralisée ou automatisée, mais bien les procédures implicites computationnelles. Par exemple, si un élève produit une seule fois l'énoncé $J$ 'ai onze ans, cela n'est certes pas suffisant pour en faire un automatisme; il importe que ce genre d'énoncé soit produit à plusieurs reprises dans différentes situations.

Quant à la compétence implicite,

- elle s'acquiert de manière incidente (sans que l'attention ne porte sur ce qui est intériorisé);

- elle est emmagasinée implicitement (elle n'est pas disponible à la conscience);

- et elle est utilisée automatiquement (sans contrôle conscient).

C'est ce qui constitue la grammaire interne de l'élève. C'est pourquoi il importe ici de se référer à la manière dont l'enfant acquiert sa langue première : pour apprendre une L2/LE, il faut commencer par apprendre à parler, c'est-à-dire développer une compétence implicite, une grammaire interne, vraisemblablement constituée, comme on l'a mentionné ci-dessus, de connexions neuronales fréquentes. C'est pourquoi on ne saurait enseigner une L2/LE comme on enseigne les autres matières scolaires, comme s'il s'agissait d'une matière scolaire comme les autres.

C'est la «pratique fonctionnelle de la langue », c'est-à-dire l'utilisation de la langue, et non la connaissance explicite de règles, qui permet de développer la compétence implicite, c'est-à-dire l'utilisation de la langue dans des situations authentiques de communication (Paradis, 1994, 2004 et 2009). C'est donc cette pratique fonctionnelle de la langue qui peut permettre d'améliorer l'apprentissage procédural. Il s'agit de la pratique d'énoncés, en situation authentique de communication. Ce qui est pratiqué est donc un processus plutôt qu'un savoir sous forme de règles. Par exemple, sur le plan phonétique, même si un locuteur est conscient des sons qu'il veut produire, il reste qu'il ne peut être conscient que du produit ou du résultat obtenu (tel ou tel son), et non du processus pour y parvenir : il n'est pas conscient de la position et du mouvement des organes phonatoires. En morphosyntaxe, le phénomène est relativement semblable : s'il y a eu apprentissage conscient de règles de grammaire, ce qui est emmagasiné dans la mémoire est la formulation de ces règles. Ce qui est automatisé n'est pas la connaissance explicite de ces règles (ou leur formulation), mais bien leur application ou l'application de tout autre processus encore mal connu. Sur le plan lexical, toutefois, tout porte à croire qu'il faille distinguer entre le plan de l'acquisition et le plan de l'usage. En effet, alors que l'acquisition du vocabulaire paraît consciente, l'utilisation de ces mêmes mots en contexte paraît plutôt être automatique : nous ne sommes pas conscients des mécanismes de sélection des mots dans la formulation d'un énoncé (Paradis, 1994 et 2004).

Quant à la grammaire externe, elle devrait être réservée à l'apprentissage conscient des règles de l'écriture, spécifiques à la langue écrite, ce qui fait appel à un processus d'apprentissage différent. Cela se comprend puisque, en lisant ou en écrivant, il est possible de faire un retour sur sa langue, de s'arrêter, en 
quelque sorte, afin de «monitoriser » la langue produite, ce qui peut difficilement être le cas lors de la production orale : l'auto-analyse de ses productions orales ne peut que freiner l'aisance à communiquer. Par exemple, comment pourrait-on arriver à communiquer oralement avec aisance en tentant « d'appliquer» les règles de la place de l'adjectif épithète en français? L'application de ce genre de règles ne pourrait que freiner ou bloquer toute spontanéité. En règle générale, pour l'enseignement de l'oral, contrairement à une opinion largement répandue, le recours à un enseignement explicite de la grammaire n'est pas approprié. Quant à la correction des erreurs, elle joue un rôle crucial, à condition que l'élève utilise et réutilise en contexte authentique de communication les phrases corrigées.

\subsection{Une conception véritablement communicative de la langue}

Comme nous l'avons vu précédemment, la conception que se font les enseignants de l'acquisition d'une L2/LE a un impact sur la conception qu'ils se font de la langue. Or, l'avènement de l'approche communicative, au milieu des années soixante-dix, n'a malheureusement pas été accompagné, comme cela aurait dû être le cas, d'un véritable changement en profondeur de la conception de la langue. Et même si tous les écrits de l'époque traitent de la langue comme moyen de communication, il semble bien qu'il ne s'agisse que d'un changement cosmétique, superficiel, d'ordre terminologique. En effet, comme on l'a vu ci-dessus, la langue est toujours effectivement traitée comme un objet d'études scolaires qu'il faut faire acquérir avant de pouvoir l'utiliser en tant que véritable moyen de communication. Il est pourtant établi que, d'une part, le savoir n'est pas nécessaire pour apprendre à communiquer oralement et que, d'autre part, le savoir ne peut se transformer en habileté. C'est plutôt la fréquence des connexions neuronales qui est nécessaire, de manière à ce que l'élève parvienne à penser dans la L2/LE plutôt qu'à traduire (Ellis, 1994; Paradis 2004 et 2009). En d'autres mots, on ne peut transformer un objet de connaissance en moyen de communication, par exemple, à l'aide d'activités ou d'exercices associés à des apprentissages centrés sur la langue (focus on form). C'est d'ailleurs ce qui explique le succès des classes d'immersion, dans la mesure où, dès les débuts de l'apprentissage, l'accent est mis sur l'utilisation même de la langue en salle de classe plutôt que sur l'apprentissage d'un savoir explicite sur la langue. Pour enseigner à communiquer dans une L2/LE, en milieu scolaire, il importe donc de mettre au point des stratégies d'enseignement de la langue qui évitent de passer par le détour d'un savoir explicite. Autrement dit, il importe de recourir à des stratégies d'enseignement qui ont pour but de développer directement l'habileté à communiquer, sans passer par le détour d'un savoir. C'est ce que permet la référence à la théorie neurolinguistique du bilinguisme de Paradis.

Selon la théorie de Paradis, donc, une langue est considérée avant tout comme un véritable moyen de communication et non comme un objet d'étude grammaticale ou comme un moyen de description du réel. En visant au développement direct de l'habileté à communiquer, sans passer par le détour d'un savoir, la langue peut être acquise par l'élève afin de lui permettre d'exprimer ses idées et ses sentiments en fonction de ses propres désirs de communication dans des situations authentiques. Ainsi, en évitant de passer par le détour d'un savoir, une langue peut être acquise plus rapidement et, grâce à la correction des erreurs commises par les élèves, être utilisée correctement de manière spontanée et indépendante, sans qu'il soit nécessaire de recourir à la grammaire externe (sauf pour l'apprentissage de certains aspects de l'écriture).

\subsection{Vers un enseignement véritablement communicatif d'une L2/LE}

Ainsi, comme on l'a vu ci-dessus, la capacité de communiquer spontanément dans une langue repose sur le développement non conscient d'une compétence implicite de la langue. Et, pour parvenir à automatiser (ou " procéduraliser ») les structures langagières tout en se concentrant sur le message, c'est-à-dire à les « intérioriser» pour développer sa grammaire interne (une compétence implicite), l'élève doit les utiliser et les réutiliser à plusieurs reprises à l'oral en diverses situations. Pour l'enseignement de l'oral, il serait donc inapproprié de commencer par l'acquisition d'un savoir explicite, qui prend beaucoup de temps et qui n'est pas nécessaire. 
C'est ainsi que, forts de cette conception de l'acquisition et de la nature d'une langue, nous avons mis au point et implanté un nouveau régime pédagogique il y a maintenant plus d'une décennie, en 1998, selon une approche que nous qualifions de «transdisciplinaire » : le français intensif (FI). Le FI s'adresse à des élèves de $5^{\mathrm{e}}$ ou de $6^{\mathrm{e}}$ année du système scolaire canadien, c'est-à-dire à des élèves de 10 ou 11 ans (l'équivalent, dans le système scolaire français, du CM1 et du CM2). On augmente alors considérablement le nombre d'heures consacrées à l'enseignement du FLS : par exemple, le nombre d'heures peut passer de 90 à environ 300 ou même à 350 heures/année, réparties sur les cinq premiers mois d'une seule année scolaire. Il faut donc réduire de moitié le nombre d'heures consacrées à l'apprentissage des matières scolaires comme l'anglais langue première, les sciences, les sciences humaines et sociales, l'éducation à la santé, etc. (sauf les mathématiques, ainsi que l'éducation physique et la musique). Le FI repose avant tout sur l'étude comparée des processus cognitifs sous-jacents à l'apprentissage de la langue première (L1) et des diverses matières scolaires et, en particulier, du FL2/LE.

Dans le cadre de ce régime pédagogique, nous avons développé quatre stratégies d'enseignement du FLS qui découlent de la théorie de Paradis, et qu'il convient maintenant de présenter succinctement (pour plus de détails, cf. Netten et Germain, 2007; Germain et Netten, 2009; Germain et Netten, à paraître).

\section{INPUT : modéliser une ou des phrases authentiques liées au thème}

À l'instar de nombreux chercheurs dans le domaine, nous sommes d'avis que pour apprendre une L2/LE à des fins de communication, il faut un input et un output (Swain, 1985). Et, pour notre part, nous avons pris soin de mettre au point des stratégies d'enseignement propres à chacun de ces concepts, de manière à établir un lien très étroit entre la théorie et la pratique. L'input consiste en un modèle, donné par l'enseignant, de phrases complètes en rapport avec une situation authentique de communication. Cela signifie que l'élève est exposé, dès le début, à des phrases qui ont un sens pour lui. En faisant commencer toute unité pédagogique par la modélisation, l'accent est mis sur le principe d'authenticité de la communication. Cela permet de renforcer cette conception qu'une langue sert avant tout à communiquer des messages personnels et authentiques. Le principe d'authenticité signifie qu'une langue que l'on apprend, même en milieu scolaire, est bien plus qu'une longue liste de mots de vocabulaire, isolés de leur contexte, de dialogues mémorisés ou de règles de grammaire abstraites à faire mémoriserr. Par langue authentique, nous entendons l'utilisation de phrases, c'est-à-dire de structures langagières signifiantes, en contexte réel.

Par exemple, s'il s'agit de parler des animaux domestiques, à l'aide de gestes ou même d'une illustration, l'enseignant pourra dire : J'ai un chien. Il s'appelle... (l'enseignant donne le véritable nom de son chien). S'il n'a pas de chien, il pourra alors parler du chien d'un ami ou d'un parent; ou d'un chat ou d'un oiseau, le cas échéant. Il s'agit pour l'élève d'apprendre à faire la conversation dans la L2. Ainsi, l'enseignant donne à l'élève un modèle langagier lui permettant d'entamer une conversation sur un animal domestique, par exemple (ou sur sa famille, et ainsi de suite). On ne commence donc pas par poser des questions à l'élève, comme il est proposé dans la plupart des manuels, car l'élève serait incapable de répondre, sans avoir été exposé préalablement à un modèle langagier lui permettant de formuler sa réponse personnelle. L'apprentissage de la L2/LE commence donc par le développement implicite de l'habileté orale plutôt que d'un savoir explicite sur la langue (pour plus de détails, cf. Netten et Germain, 2009). Au fur et à mesure que l'élève progresse dans son apprentissage de la langue, bien entendu, ce sont des phrases de plus en plus complexes qui lui sont proposées, par exemple : Dans ma vie, une personne importante pour moi est... parce qu'elle m'a enseigné à....

\section{OUTPUT : faire utiliser et réutiliser les phrases modélisées, en les adaptant}

Comme il s'agit de donner à l'élève des habitudes langagières nouvelles, à l'oral, c'est donc l'utilisation fréquente, par l'élève, de phrases authentiques modélisées, adaptées à ses propres besoins de communication, qui correspond à l'output langagier nécessaire à l'acquisition d'une compétence implicite en L2/LE. Dans cette perspective, il est alors important que les phrases modélisées par l'enseignant et 
adaptées à la situation personnelle de l'élève ne soient pas seulement "répétées », isolées de leur contexte, comme c'était le cas dans les méthodes audio-orales. On vise donc au développement d'une « automaticité créatrice 》 (Gatbonton et Segalowitz, 2005), c'est-à-dire à ce que l'élève puisse " créer » avec la langue de manière à pouvoir faire de nouvelles combinaisons avec divers éléments déjà appris, afin d'exprimer de nouvelles idées ou de nouveaux sentiments. Ainsi, afin de parvenir à créer des patterns dans la tête de l'élève, c'est-à-dire des réseaux de connexions neuronales, l'output langagier se présente successivement sous diverses formes :

1. questionner les élèves pour faire utiliser et réutiliser les phrases modélisées, en les adaptant

Pour faire utiliser et réutiliser la langue en classe, l'enseignant multiplie les occasions de poser de nombreuses questions aux élèves. Les questions permettent aux élèves d'utiliser et de réutiliser, en situation authentique de communication, les phrases apprises à partir des modèles donnés par l'enseignant. Pour reprendre un des exemples donnés ci-dessus, si l'enseignant a donné aux élèves le modèle langagier suivant, concernant les animaux domestiques: Moi, j'ai un chien, il peut alors enchaîner en posant la question : Et toi, est-ce que tu as un chien? (le cas échéant : un chat? un oiseau?) Comment s'appelle-t-il?, etc. Les mots de vocabulaire dont l'élève a besoin pour communiquer ses messages lui sont alors donnés oralement, sur demande, par l'enseignant, en contexte authentique. Ainsi, si un élève qui veut dire qu'il a un lapin à la maison dit: Moi, j'ai... comment dit-on "rabbit »?, l'enseignant dit: Un lapin, à la suite de quoi l'élève sera incité à produire la phrase complète: Moi, j'ai un lapin. Bien entendu, toujours en vertu du principe d'authenticité, l'enseignant veillera à présenter également et à faire utiliser certaines formes négatives utiles: Je n'ai pas de chien. On notera que le fait d'intégrer tout mot de vocabulaire nouveau dans une phrase réelle montre bien qu'une langue ne sert pas avant tout à décrire, comme il est proposé dans la plupart des manuels (Qu'est-ce que c'est? - C'est...), mais bien à communiquer.

À cet égard, il est à noter que l'enseignant est appelé à ne modéliser qu'un nombre restreint de mots de vocabulaire, toujours en contexte. C'est d'ailleurs pour cette raison que la stratégie d'enseignement de la modélisation est préférée à celle du remue-méninges car, au commencement d'une leçon ou d'une unité pédagogique, le remue-méninges ne conduit, la plupart du temps, qu'à une longue énumération, le plus souvent en anglais, de mots de vocabulaire isolés de leur contexte. La modélisation, de son côté, présente l'avantage de donner à l'élève, dès le début, des phrases qu'il est appelé à utiliser, en les adaptant, pour transmettre des messages authentiques.

Les phrases modélisées sont donc reproduites en étant adaptées à la situation personnelle des élèves, de manière à bien montrer qu'une langue est effectivement un moyen de communication à l'aide de phrases authentiques. Du coup, les élèves sont plus impliqués cognitivement dans leur apprentissage, car il ne s'agit pas simplement pour eux de répéter des phrases toutes faites ou d'apprendre à réciter par cœur des dialogues, mais bien de reprendre des phrases modélisées, en les adaptant à leur situation personnelle, afin de pouvoir exprimer ce qu'ils veulent dire. Il faut donc parvenir à faire penser les élèves dans la L2/LE.

2. faire se questionner les élèves entre eux en faisant adapter leur réponse à leur situation personnelle

Si l'on reprend l'exemple de la discussion sur les animaux domestiques, l'enseignant prépare d'abord les élèves en modélisant les phrases ainsi que le mode d'interaction. Puis, il demande à des élèves de questionner d'autres élèves de la classe (de manière à s'assurer que les élèves posent correctement la question) et demande de répondre avec de légères variantes, tout en donnant un modèle de la tâche à accomplir au cours de l'étape suivante. 
3. faire interagir simultanément les élèves, en binômes, pour faire réutiliser, en les adaptant, la question et la réponse modélisées

En binômes, les élèves entament une conversation, en utilisant la question et la réponse qui viennent d'être modélisées mais, en les adaptant à leur réalité. Dans un temps donné (par exemple, 10 ou 12 secondes - pour ne pas leur laisser le temps de recourir à leur L1), les élèves se posent mutuellement ce genre de questions et y répondent en les adaptant, par exemple : As-tu un chien? (un chat? etc.) et enchaînent avec une autre question, par exemple, Comment s'appelle-t-il? Puis, les élèves changent de partenaire et, de nouveau, se posent mutuellement des questions de cette nature et y répondent.

4. questionner les élèves pour leur faire réutiliser, en les adaptant, les réponses des autres élèves.

Pour donner aux élèves une nouvelle occasion d'utiliser la langue, l'enseignant leur pose des questions sur ce que leur partenaire vient de dire, par exemple : Quel est l'animal favori de Kelly? Les élèves répondent en faisant référence aux réponses personnelles données antérieurement par leur partenaire. Et l'enseignant enchaîne en posant une nouvelle question à partir de la réponse donnée ou en faisant un commentaire, sous forme de conversation authentique. Lorsque les élèves sont familiers avec cette stratégie, ils peuvent eux-mêmes y recourir. Les enseignants peuvent également stimuler l'écoute des élèves en salle de classe en posant des questions à quelques élèves afin de vérifier leur " intention d'écoute » en les interrogeant sur le contenu des réponses données beaucoup plus tôt (parfois, au cours des jours précédents) par d'autres élèves.

En outre, pour des débutants dans l'apprentissage d'une L2, ces deux dernières étapes (3 et 4) sont répétées, dans la plupart des cas, de manière à permettre de nouvelles interactions, notamment entre élèves, afin de s'assurer de donner de nouvelles habitudes langagières.

\section{AISANCE : inciter à faire des liens}

Le concept d'aisance langagière est relativement flou et difficile à cerner, comme l'ont déjà reconnu Koponen et Riggenbach (2000), tout comme Brumfit (1984) l'avait fait il y a quelques années. En effet, pour la plupart des auteurs, il s'agit d'un phénomène d'ordre phonétique se rapportant aux difficultés avec lesquelles une personne communique : hésitations, pauses et reprises lors d'une communication orale (Faerch et al., 1984; Palmer, 1917 et 1921; Towell et al., 1996; Wood, 2001). Par contre, pour certains autres auteurs, l'aisance est parfois définie comme un phénomène plutôt global, se rapportant à la facilité avec laquelle une personne communique, notamment quant à la rapidité du débit, c'est-à-dire au nombre de syllabes produites dans un laps de temps donné (Fillmore, 1979; Hedge, 1993; Leeson, 1975; Lennon, 2000). Pour notre part, nous définirons l'aisance à communiquer comme l'habileté à mettre en relation avec facilité les diverses composantes (grammaticale et discursive, d'une part, fonctionnelle et socioculturelle, d'autre part) d'un énoncé dans une situation authentique de communication. Dans cette perspective, nous considérons les hésitations, les pauses, les allongements vocaliques, les répétitions de mots ou de syllabes, etc. comme la simple manifestation externe d'un phénomène plus complexe sousjacent : l'habileté à faire des liens, avec plus ou moins de facilité, entre les diverses composantes d'un énoncé en situation authentique de communication.

Pour que l'élève puisse communiquer oralement avec aisance, il doit tout d'abord développer sa grammaire interne, c'est-à-dire établir dans sa tête des réseaux de connexions neuronales par une utilisation fréquente de certaines formes langagières, tout en se concentrant sur le message. L'aisance est l'habileté à faire ces connexions neuronales avec spontanéité, sans hésitations. Or, comment l'élève pourrait-il parvenir à faire rapidement ces connexions, sur le plan morphosyntaxique (ou « horizontal»), s'il n'a appris que des savoirs sur la langue qui se situent en quelque sorte sur le plan " vertical », sous la forme, par exemple, de listes de mots de vocabulaire hors contexte ou de conjugaisons de verbes? C'est pourquoi, afin d'amener l'élève à faire rapidement des connexions " horizontales » dans sa tête, l'enseignant doit recourir à une pédagogie de la phrase, c'est-à-dire toujours faire produire des phrases complètes. De cette manière, l'élève sera amené à automatiser les structures langagières utilisées. En 
encourageant l'élève à ne produire que des phrases complètes, on l'amène, en quelque sorte, à bâtir sa grammaire interne : par exemple, à la question Quel âge as-tu?, l'enseignant n'acceptera pas une simple réponse comme Onze. L'élève devra produire une phrase complète, comme J'ai onze ans. Ainsi, l'élève pourra parvenir, graduellement, à établir facilement et rapidement des liens entre le message à transmettre et la structure langagière correspondante, et entre les éléments langagiers eux-mêmes, sur tous les plans : phonologique, morphologique, etc. (Netten et Germain, 2005).

\section{PRÉCISION : corriger les erreurs et faire utiliser à plusieurs reprises les phrases corrigées}

Le concept de précision est le plus souvent intuitivement compris comme l'absence d'erreurs d'ordre linguistique que fait un apprenant au moment où il tente d'utiliser la L2/LE, tant en situation décontextualisée (dans le cadre d'un exercice scolaire artificiel) qu'en situation authentique de communication; souvent, il est aussi défini comme la connaissance adéquate que possède une personne des unités et des règles de fonctionnement de la langue. Mais, dans les deux cas, la précision n'est comprise que comme un savoir sur la langue. Pour notre part, contrairement aux idées reçues, nous définirons donc la précision langagière comme pouvant désigner à la fois un savoir et une habileté. En tant que savoir, la précision consiste en la connaissance adéquate (consciente) que possède une personne des unités et des règles de fonctionnement d'une langue (composante langagière) ainsi que des règles d'usage de cette langue (composante pragmatique). En tant qu'habileté, la précision langagière consiste en la capacité que possède une personne d'utiliser correctement, de manière non consciente, les unités et les règles de fonctionnement d'une langue (composante langagière) ainsi que les règles d'usage de cette langue (composante pragmatique) dans une situation socioculturelle de communication.

Comme, d'après la théorie de Paradis, un savoir ne peut se transformer en habileté, il faut faire commencer l'apprentissage de la langue par le développement de la précision langagière en tant qu'habileté à communiquer, c'est-à-dire par l'utilisation correcte de la langue lors d'une conversation spontanée, même en salle de classe; ce qui n'est pas l'usage courant qui consiste à développer la précision langagière en tant que savoir, sous forme, par exemple, de règles de grammaire à apprendre ou de verbes à conjuguer. En effet, la correction par l'enseignant d'une phrase mal formée, suivie de la réutilisation immédiate par l'élève de la phrase corrigée (Lyster, 1998), et puis de l'utilisation à plusieurs reprises des phrases corrigées, en situation authentique de communication, vise au développement d'une langue correcte qui soit la plus conforme possible à la langue cible : c'est ce qui constitue la précision langagière, en tant qu'habileté à communiquer, par laquelle nous faisons commencer l'apprentissage de la langue. Comme il s'agit de développer d'abord une habileté, ou compétence implicite, on préférera donc recourir à la correction des erreurs plutôt qu'à l'enseignement de la grammaire. La correction des erreurs a aussi le mérite de se situer sur le plan de la phrase et, par conséquent, de développer une grammaire interne correcte (Netten et Germain, 2005). En début d'apprentissage d'une L2, l'élève peut certes imiter ou reproduire relativement correctement les modèles langagiers entendus, donnés par l'enseignant. Mais, lorsque l'élève tente de créer ses propres messages, le risque d'erreurs est plus élevé. C'est pourquoi la correction des erreurs par l'enseignant est cruciale pour que l'élève ne continue pas à développer une compétence linguistique fautive, ni à construire sa grammaire interne sur des patterns langagiers erronés (Shehadeh, 2003).

Bien entendu, les stratégies d'enseignement présentées ci-dessus se déroulent exclusivement à l'oral : rien n'est écrit au tableau. On apprend à parler en parlant, de manière à développer une compétence implicite. Par ailleurs, l'apprentissage de la L2/LE ne saurait consister simplement qu'à apprendre à communiquer oralement. Dans une perspective du développement de la littératie, il importe de mentionner l'importance de la lecture et de l'écriture. Avec la lecture des textes, débute le développement d'un savoir explicite sur la langue. L'enseignement de la lecture dans une L2 requiert des stratégies spécifiques d'enseignement, qu'il serait trop long de présenter ici (pour plus de détails, cf. Germain et Netten, 2009). L'élève doit apprendre à dégager le message d'un texte écrit. Il s'agit là aussi d'une forme de communication des idées. La lecture renforce ce que l'élève a appris à l'oral et lui permet d'accroître son vocabulaire. Elle procure aussi un savoir sur la langue et contribue à l'amélioration de la production orale et écrite. Quant à 
la lecture à haute voix, elle permet d'améliorer la prononciation, l'élocution et l'intonation, de comprendre le texte et de vérifier le rapport entre son et graphie. La lecture stimule également les conversations et les discussions, et offre une variété d'activités langagières. En tant qu'habileté, la lecture exige d'être pratiquée régulièrement et systématiquement, c'est-à-dire tous les jours. Lire implique que l'on re-connaisse à l'écrit ce que l'on peut déjà dire, ce qui pose tout le problème des rapports entre l'oral et l'écrit.

Quant à l'enseignement de l'écriture, c'est-à-dire le savoir explicite sur la langue, il n'intervient qu'à la suite du développement de la compétence implicite, même si cela peut se produire au cours d'une même journée (Germain et Netten, 2005). Dans les débuts de l'apprentissage d'une L2/LE, les élèves sont encouragés à n'écrire que ce qu'ils peuvent d'abord produire oralement. Toutefois, contrairement à certaines pratiques courantes, il ne saurait être ici question de faire écrire des phrases isolées, décousues, sans lien entre elles. Toute activité d'écriture consiste à rédiger un ou des paragraphes, c'est-à-dire une séquence de phrases liées les unes aux autres. Comme ces textes, si brefs soient-ils, traitent de messages personnalisés, même dans le cadre d'une thématique commune (par exemple, leur nourriture préférée), ils peuvent donc être lus avec grand intérêt par les autres élèves de la classe. Enfin, afin de réduire le nombre d'erreurs morphosyntaxiques dans leurs textes écrits, les enseignants incitent les élèves à utiliser des énoncés qu'ils peuvent déjà produire oralement de manière correcte : par exemple, comment pourrait-on penser qu'un élève puisse écrire $J$ 'ai onze ans si, à l'oral, cet élève produit, sur le calque de l'anglais, l'énoncé fautif Je suis onze. La correction des erreurs à l'oral ne peut que contribuer à l'amélioration des productions écrites de l'élève. Dans une perspective du développement de la littératie, à laquelle nous adhérons, il importe de développer chez l'élève, tant à l'oral qu'à l'écrit, des habiletés à communiquer des messages authentiques et signifiants pour lui.

Toutefois, contrairement à ce que nous avons observé dans de nombreuses classes de langue, on ne saurait présupposer que l'élève sait lire et écrire dans la L2/LE sous prétexte qu'il sait déjà lire et écrire dans sa langue première. Ce n'est pas parce qu'un élève sait lire et écrire dans sa langue première qu'il est automatiquement en mesure de lire et d'écrire dans la L2/LE. En effet, l'apprentissage d'une autre langue fait appel à un système différent de correspondance des sons et de la graphie : par exemple, en français, il $\mathrm{y}$ a de nombreuses façons d'écrire, selon le contexte, le son 'o' : eau, haut, $o, \hat{o}$, etc. On ne saurait donc présupposer que l'élève n'a qu'à transférer dans la L2/LE ses habiletés déjà acquises en lecture dans sa langue première. Il en va de même de l'écriture. Mais, tant en lecture qu'en écriture, on ne saurait faire l'économie de l'acquisition préalable de l'oral.

Ainsi, les stratégies d'enseignement véritablement communicatives que nous préconisons reposent sur une distinction très nette entre l'ordre d'acquisition d'un savoir et d'une habileté; entre l'ordre d'acquisition de la grammaire interne et de la grammaire externe; et entre l'ordre d'acquisition de l'oral et de l'écrit. Il s'agit donc d'un changement majeur de stratégies d'enseignement non véritablement communicatives de l'oral, le plus souvent utilisées dans le cadre du régime pédagogique du français de base au Canada.

\section{Résultats à l'oral en français intensif (FI)}

Afin de mesurer l'efficacité du FI (comme dans le cas du français de base), nous avons procédé à l'évaluation de ce nouveau régime pédagogique dans la plupart des provinces et territoires canadiens, à partir de 2003. À cette fin, le même test d'entrevue orale individuelle qui avait été administré comme prétest aux élèves en français de base, a été administré comme post-test à plus de 600 élèves de $5^{\mathrm{e}}$ année en $\mathrm{FI}$, après environ 300 heures intensives réparties sur cinq mois. Comparativement aux résultats obtenus en français de base, ceux du FI semblent prometteurs puisque plus de $70 \%$ des élèves atteignent le niveau Basic Low, c'est-à-dire qu'ils peuvent communiquer avec une certaine spontanéité, avec des phrases complètes (Figure 3). 
Figure 3 - Français intensif: production orale, niveau atteint après 5 mois (300 heures intensives), $5^{\mathrm{e}}$ année (élèves : 11 ans), 32 classes, 2003-2006 $(\mathrm{N}=655)$

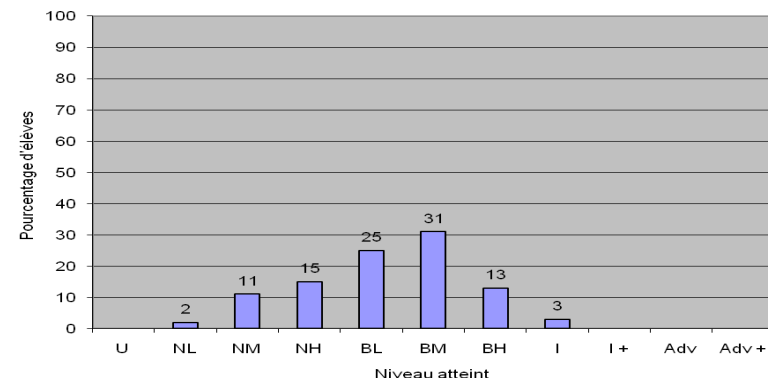

En FI, les résultats obtenus sont dus vraisemblablement en grande partie au fait que ce régime pédagogique ne comporte pas les trois confusions dont il a été question ci-dessus : on développe directement la compétence implicite de l'élève sans passer par le détour d'un savoir explicite. De plus, on aide l'élève à développer sa grammaire interne plutôt que de passer inutilement du temps à faire apprendre les règles de la langue pour le développement de l'oral. Aussi, on passe de l'oral à l'écrit, et non l'inverse. Enfin, le mode de progression suivi va du développement d'une compétence implicite à un savoir explicite au sujet d'une langue que l'on peut déjà utiliser spontanément. Concrètement, afin de rendre compte des résultats obtenus, il faut se référer à l'utilisation des quatre stratégies d'enseignement de l'oral qui ont été présentées ci-dessus. Certes, le nombre d'heures d'enseignement et l'intensité sont deux facteurs importants, mais il semble bien que c'est le recours aux stratégies d'enseignement utilisées qui est le facteur le plus déterminant pour le développement d'une compétence implicite. C'est, en tout cas, ce que montrent quelques études de cas que nous avons faites sur le sujet et qui ont été rapportées ailleurs (Netten et Germain, 2005). Nous nous permettons de ne reproduire ici, succinctement, qu'une de ces études de cas.

Cette étude implique une enseignante et ses deux classes de $5^{\mathrm{e}}$ année d'une trentaine d'élèves chacune (âge : 10 ans), au cours de deux années non consécutives (2002-2003 : 372 heures intensives et 20042005 : 317 heures intensives), dans la même école. D'après les résultats des pré-tests et des post-tests, après cinq mois d'apprentissage intensif du français au cours de la première année (en 2002-2003), les élèves n'ont progressé que d'un niveau et demi (de 11,4 à 12,7), alors qu'au cours de la deuxième année (2004-2005), ils ont progressé de plus de trois niveaux (de 11,8 à 15,1) sur l'échelle MSS utilisée. Les résultats obtenus la deuxième année ne peuvent certainement pas s'expliquer uniquement par le nombre d'heures intensives, alors qu'il y a eu de fait une réduction de 55 heures d'enseignement la deuxième année. En effet, d'après nos observations de classe, les résultats ne peuvent vraisemblablement s'expliquer que par le changement radical, chez l'enseignante, de ses stratégies d'enseignement, suite à son inscription à un stage sur le FI suivi au cours de l'été qui a précédé la deuxième année d'enseignement.

Il ressort de ce qui précède qu'un changement de paradigme s'impose si on veut parvenir à construire une didactique des langues qui contribue effectivement à faire communiquer les élèves dans leur L2/LE. Tant que sera négligée, comme cela est présentement le cas, l'étude du fondement psychologique de l'apprentissage ou de l'acquisition d'une L2/LE, la didactique des langues sera condamnée à piétiner. Or, à cet égard, en dépit de ses nombreux mérites, il semble que l'on soit loin d'un changement de paradigme dans le récent Cadre européen commun de référence pour les langues (2000), en dépit des affirmations contraires de Richer (2009). Même si le chapitre 6 est consacré spécifiquement aux relations entre l'enseignement et l'apprentissage, les grands débats actuels chez les acquisitionnistes, ainsi que les concepts clés de l'acquisition d'une L2/LE, comme ceux de compétence implicite et de savoir explicite, en sont absents. En ce sens, le CECR paraît se situer nettement dans la tradition d'une perspective avant tout curriculaire, ce qui ne peut que contribuer à perpétuer le sérieux déséquilibre dont souffre depuis plusieurs années la didactique des langues (Germain, 2001) dans le développement de ses dimensions 
linguistique, psychologique et pédagogique. Devant ce constat, ne serait-il pas temps de rétablir un certain équilibre et une certaine cohérence entre les principaux plans de la didactique des langues?

Ainsi, nos nombreuses observations de classe ont mis en évidence l'inadéquation entre le but visé (l'habileté à communiquer) et les moyens adoptés (des stratégies d'enseignement non véritablement communicatives ) pour atteindre ce but. Et comment expliquer cette inadéquation? À notre avis, une explication serait que le choix des stratégies d'enseignement est déterminé à la fois par la conception qu'on se fait de l'acquisition de la langue et par la conception de la langue qui en découle. Donc, schématiquement, cette conception pourrait être ainsi représentée (voir Graphique 1).

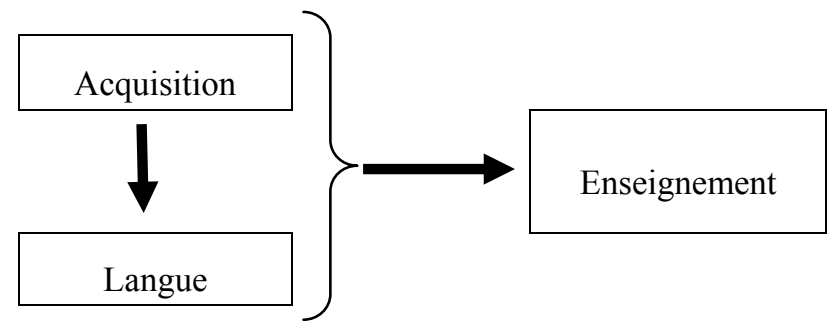

Graphique 1 - Relations entre les conceptions de l'acquisition d'une L2/LE, de la nature d'une L2/LE et de son enseignement

En nous plaçant sur le plan plus général de la didactique des langues, il ressort que le facteur premier, le plus déterminant, de toute stratégie d'enseignement, serait d'abord la conception (implicite ou explicite) que l'on se fait du mode d'acquisition d'une L2/LE, d'où découle la conception de la langue. De manière générale, dans la perspective de la didactique des langues, c'est le plan psychologique (psychologie de l'acquisition ou de l'apprentissage) qui serait fondamental et déterminerait, à l'insu des gens dans la plupart des cas, le plan linguistique (la conception de la langue) et ces deux plans, psychologique et linguistique, détermineraient à leur tour le plan spécifiquement pédagogique (les stratégies d'enseignement - voir Graphique 2).

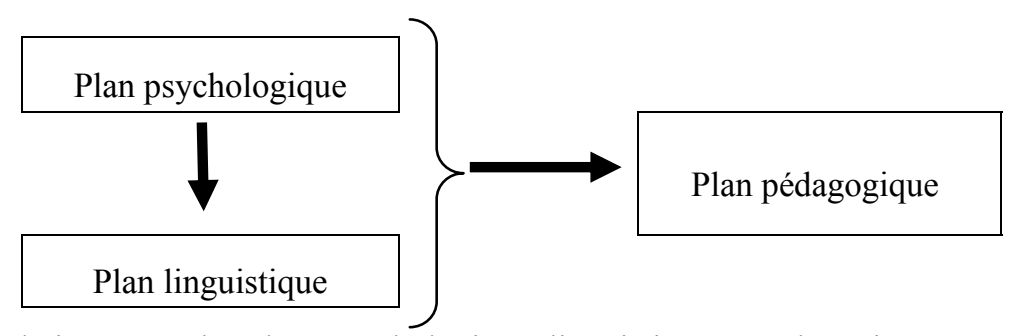

Graphique 2 - Relations entre les plans psychologique, linguistique et pédagogique

\section{CONCLUSION}

Comme nous l'avons exposé dans cet article, les stratégies d'enseignement utilisées en classe de L2/LE sont influencées par la conception implicite de la langue que se font les enseignants, laquelle découle ellemême de la conception qu'ils ont de l'acquisition de la langue. Ces considérations permettent de faire voir sous un nouvel angle le domaine complexe des relations entre le plan psychologique (la conception de l'acquisition d'une L2/LE) et les plans linguistique (la conception de la langue) et pédagogique qui en découlent (par exemple, les stratégies d'enseignement). Il ressort donc de ce qui précède que la souche de cette filiation serait la conception que l'on se fait du mode d'acquisition d'une L2/LE : en effet, faut-il faire acquérir d'abord des savoirs sur la langue afin de les transformer par la suite en habiletés à communiquer, suivant une opinion largement répandue, ou, à l'inverse, faire acquérir d'abord des habiletés à communiquer, d'après la théorie de Paradis, sans passer par le détour des savoirs? C'est la 
manière dont on répond à cette question qui détermine le choix des stratégies d'enseignement qu'on utilise en salle de classe. Il s'agit d'une hypothèse sur le sens des relations entre ces trois plans dont les retombées seraient d'une grande importance : en effet, l'étude du fondement psychologique, notamment neurolinguistique, de l'acquisition d'une L2/LE devrait occuper la première place dans tout programme de formation ou de perfectionnement des enseignants, en raison de son impact.

\section{Références bibliographiques}

Anderson, J.R. (1990). Cognitive Psychology and Its Implications. $3^{\mathrm{e}}$ éd., New York: W.H. Freeman.

Brumfit, C. (1984). Communicative Methodology in Language Teaching: The Roles of Fluency and Accuracy. Cambridge : Cambridge University Press.

Cadre européen commun de référence pour les langues (2000). Didier et Conseil de la Coopération culturelle Comité de l'éducation, Division des langues vivantes, Strasbourg.

Dekeyser, R. (1998). Beyond focus on form: Cognitive perspectives on learning and practicing second language grammar. In Doughty, C. et Williams, J. (éds). Focus on Form in Classroom Second Language Acquisition, Cambridge : Cambridge University Press.

Ellis, N.C. (2005). At the interface : Dynamic interactions of explicit and implicit language knowledge. Studies in Second Language Acquisition, $N^{\circ} 27,305-352$.

Ellis, N.C. ( 2002). Frequency Effects in Language Processing. Studies in Second Language Acquisition, $n^{\circ} 24,143-$ 188.

Ellis, N.C. (1994). Implicit and explicit processes in language acquisition: An introduction. In Ellis, N.C. (éd.), Implicit and explicit learning of languages, San Diego, CA : Academic Press, 1-32.

Faerch, C., Haastrup, K.I. et Phillipson, R. (1984). Learner Language and Language Learning. Clevedon: Multilingual Matters.

Fillmore, C.J. (1979). On fluency. In Fillmore, C.J., Kempler, D. et Wang, W.S-Y. (éds.). Individual Differences in Language Ability and Language Behavior, New York : Academic Press, 85-161.

Gatbonton, E. et Segalowitz, N. (2005). Rethinking Communicative Language Teaching: A Focus on Access to Fluency. Canadian Modern Language Review/Revue canadienne des langues vivantes, Vol. 61, $n^{\circ}$ 3, 325-353.

Germain, C. (2001). La didactique des langues : une autonomie en devenir. Numéro spécial : Le Français dans le Monde, intitulé : Théories linguistiques et enseignement du français aux non francophones, Collection Recherches et applications, 13-27.

Germain, C. et Netten, J. (à paraître). Stratégies d'enseignement de la communication à l'oral en langue seconde ou étrangère. In Florilège Québec. Ouvrage collectif. Texte de la conférence prononcée à Québec, au Congrès de la FIPF (Fédération Internationale des Professeurs de Français), juillet 2008.

Germain, C. et Netten, J. (2009). Le français intensif (FI), le pré-FI et le post-FI/FA (français approfondi) Introduction. In Germain, C. et Netten, J. (éds.), Le français intensif-Guide pédagogique interprovincial, $2^{\mathrm{e}}$ éd., $1-47$.

Germain, C. et Netten, J. (2005). Place et rôle de l'oral dans l'enseignement/apprentissage d'une L2. Babylonia, $n^{\circ} 2$, 7-10.

Groulx, J.-F. (2005). Étude des relations entre l'enseignement et l'apprentissage de la précision linguistique et de l'aisance à communiquer dans cinq classes intensives de français langue seconde. Mémoire de M.A. inédit : UQAM (Université du Québec à Montreal), $191 \mathrm{p}$.

Hedge, T. (1993). Key concepts in ELT (fluency). ELT Journal, $n^{\circ}$ 47, 275-292.

Koponen, (M. et Riggenbach, H. (2000). Overview : Varying perspectives on fluency. In Riggenbach, H. (éd.), Perspectives on Fluency, Ann Arbor : The University of Michigan Press, 5-24.

Krashen, S. (1981). Second Language Acquisition and Second Language Learning. Oxford: Pergamon Press.

Leeson, R. (1975). Fluency and Language Teaching. London : Longman. 
Lennon, P. (2000). The lexical element in spoken second language fluency. In Riggenbach, H. (éd.). Perspectives on Fluency, Ann Arbor : The University of Michigan Press, 25-42.

Lightbown, P. et Spada, N. (1999). How Languages are Learned. Oxford : Oxford University Press.

Lyster, R. (1998). Recasts, repetition and ambiguity in L2 classroom discourse. Studies in Second Language Acquisition, Vol. 20, 51-81.

Nassaji, H. et Fotos, S. (2004). Current developments in research on the teaching of Grammar. Annual Review of Applied Linguistics, Vol. 24, 109-125.

Netten, J. et Germain, C. (2009). The future of intensive French in Canada. Canadian Modern Language Review/Revue canadienne des langues vivantes, Vol. 65, $n^{\circ}$ 5, 757-786.

Netten, J. et Germain, C. (2007). Learning to communicate effectively through intensive instruction in French. In M. Dooly (éd.), Teachers' Voices on Innovative Approaches to Teaching and Learning Languages, Cambridge: Cambridge Scholar Press.

Netten, J. et Germain, C. (2005). Pedagogy and second language learning : Lessons learned from Intensive French. Revue canadienne de linguistique appliquée / Canadian Journal of Applied Linguistics, $n^{\circ}$ 8.2, 183-210.

Painchaud, G. et Boyko, J. (1990). National core French study - Language Syllabus. Ottawa : Canadian Association of Second Language Teachers.

Palmer, H.E. (1921). The Principles of Language-Study. London : Harrap.

Palmer, H.E. (1917). The Scientific Study and Teaching of Languages. London : Harrap.

Pambianchi, G. (2003). Modélisation des démarches pédagogiques dans les pratiques de classe de français langue seconde chez les adultes. Ph.D. inédit : UQAM (Université du Québec à Montréal), 458 p.

Paradis, M. (2009). Declarative and Procedural Determinants of Second Languages. Amsterdam/Philadelphia : John Benjamins.

Paradis, M. (2004). A Neurolinguistic Theory of Bilingualism. Amsterdam/Philadelphia : John Benjamins.

Paradis, M. (1994). Neurolinguistic aspects of implicit and explicit memory: implications for bilingualism. In Ellis, N.C. (éd.), Implicit and Explicit Learning of Second Languages, London : Academic Press, 393-419.

Pienemann, M. (1989). Is language teachable? Psycholinguistic experiments and hypotheses. Applied Linguistics, Vol. 10, $N^{o} 1,52-79$.

Prabhu, N.S. (1987). Second Language Pedagogy. Oxford : Oxford University Press.

Richer, J.-J. (2009). Lectures du Cadre européen commun de référence pour les langues : continuité ou rupture didactique? In L'approche actionnelle dans l'enseignement des langues, Paris : Maison des langues.

Schmidt, R.W. (1990). The role of consciousness in second language learning. Applied Linguistics, Vol. 11, $N^{\circ} 2$, 129-158.

Shehadeh, A. (2003). Learner output, hypothesis testing, and internalizing linguistic knowledge. System, $n^{o} 31,155-$ 171.

Swain, M. (1985). Communicative competence: Some roles of comprehensible input and comprehensible output in its development. In Gass, S. et Madden, C.G. (éds.), Input in Second Language Acquisition, Rowley, M.A. : Newbury House.

Towell, R., Hawkins, R. et Bazergui, N. (1996). The development of fluency in advanced learners of French. Applied Linguistics, $N^{o} 1,84-119$.

Truscott, J. (1999). What's wrong with oral grammar correction. Canadian Modern Language Review/Revue canadienne des langues vivantes, Vol. $5, N^{\circ} 4,437-467$.

Van Patten, B. (2002). Processing instruction : An update. Language Learning, Vol. 52, $N^{o}$ 4, 755-803.

White, J. et Ranta, L. (2002). Examining the Interface between Metalinguistic Task Performance and Oral Production in a Second language. Language Awareness, Vol. 11, $N^{\circ}$ 4, 259-290. 
Wood, D. (2001). In search of fluency: What is it and how can we teach it?. Canadian Modern Language Review/Revue canadienne des langues vivantes, Vol. 57, 573-589.

${ }^{1}$ Deux composantes majeures de l'enseignement de la langue sont les stratégies d'enseignement et les activités pédagogiques ou didactiques. Par stratégies d'enseignement, nous entendons les gestes concrets posés par l'enseignant, en salle de classe, afin de créer les conditions propices à l'apprentissage de la matière enseignée, en l'occurrence, le FL2/FLE. Quant aux activités pédagogiques ou didactiques, et à leur séquence, elles relèvent de l'aspect curriculaire et forment, dans la majorité des cas, le contenu des cours de langue (Pambianchi, 2003). La manière dont une même activité se déroule dans une salle de classe varie en fonction des stratégies d'enseignement utilisées, qui sont d'une nature dynamique (Groulx, 2005).

${ }^{2}$ Les nombreux exemples qui suivent ont été puisés dans plusieurs manuels différents, qu'il serait trop long de citer en bibliographie, tous destinés à l'apprentissage du FL2/FLE.

${ }^{3}$ Dans le passé, certaines personnes croient avoir appris une L2/LE en procédant de cette manière. Dans les faits, il n'y a qu'environ $10 \%$ des élèves qui, en milieu scolaire, réussissent à apprendre la langue apparemment de cette manière. Mais, on oublie que la très grande majorité des élèves ont abandonné leur étude de la langue et que ceux qui ont réussi sont habituellement ceux qui étaient très motivés ou très « doués » pour l'apprentissage de la langue ou qui ont pu pleinement profiter d'un séjour linguistique à l'étranger au cours duquel ils ont eu l'occasion d'utiliser effectivement la langue qu'ils étaient en train d'apprendre.

${ }^{4}$ Pour un exposé critique relativement détaillé sur la question, voir Netten et Germain (2005).

${ }^{5}$ Il y a d'autres catégories, pour les niveaux plus avancés, mais elles ne sont pas reproduites ici car elles sont non pertinentes aux fins de nos analyses.

${ }^{6}$ À cette liste, il convient également d'ajouter les travaux de la communauté de chercheurs européens dont ont rendu compte divers numéros de la revue AILE (Acquisition et Interaction en Langue Étrangère - notamment AILE 8, 1996 et AILE 16, 2002), publiée par l'Association Encrages (université de Paris VIII). 